\title{
ON THE $C^{*}$-ALGEBRA GENERATED \\ BY THE LEFT REGULAR REPRESENTATION OF A LOCALLY COMPACT GROUP
}

\author{
ERIK BÉDOS
}

(Communicated by Palle E. T. Jorgensen)

\begin{abstract}
Let $\lambda$ denote the left regular representation of a locally compact group $G$ on $L^{2}(G)$ and $C^{*}(\lambda(G))$ the $C^{*}$-algebra generated by $\lambda(G)$. We show that the amenability of $G$ and the amenability of $G$ considered as a discrete group both may be characterized in terms of $C^{*}(\lambda(G))$.
\end{abstract}

\section{INTRODUCTION}

We first fix some notation. Throughout this note we let $G$ denote a locally compact (Hausdorff topological) group equipped with a fixed left Haar measure $\mu$ and $G_{d}$ denote the group $G$ considered as a discrete group. As usual, $L^{1}(G), L^{2}(G)$, and $L^{\infty}(G)$ are defined with respect to $\mu$. The left regular representation of $G$ on $L^{2}(G)$, defined by

$$
(\lambda(g) \xi)(h)=\xi\left(g^{-1} h\right), \quad \xi \in L^{2}(G), g, h \in G,
$$

is well known to be a (strongly) continuous unitary representation of $G$. We shall denote by $\lambda_{d}$ the left regular representation of $G_{d}$ on $l^{2}\left(G_{d}\right)$. All undefined terminology in this paper is explained in at least one of the references [5, $8,11,15,17,18]$.

Much attention has been devoted to the study of the following operator algebras associated with $G$ : the full group $C^{*}$-algebra $C^{*}(G)$, the reduced group $C^{*}$-algebra $C_{r}^{*}(G)$, and the group von Neumann algebra $v N(G)$. We recall that $C^{*}(G)$ is defined as the enveloping $C^{*}$-algebra of $L^{1}(G)$ considered as an involutive Banach algebra with an approximate identity. If $\mathscr{B}\left(L^{2}(G)\right)$ denotes the bounded linear operators on $L^{2}(G)$, then $C_{r}^{*}(G)$ is the $C^{*}$-subalgebra of $\mathscr{B}\left(L^{2}(G)\right)$ generated by the convolution operators $T_{f}, f \in L^{1}(G)$, where $T_{f}(\xi)=f * \xi, \xi \in L^{2}(G)$. At last, $v N(G)$ is the von Neumann subalgebra of $\mathscr{B}\left(L^{2}(G)\right)$ generated by $\lambda(G)=\{\lambda(g), g \in G\}$ or, equivalently, $v N(G)=$ $\lambda(G)^{\prime \prime}=C_{r}^{*}(G)^{\prime \prime}$, where " denotes the double commutant (in $\mathscr{B}\left(L^{2}(G)\right)$ ).

Received by the editors May 18, 1992; this paper was presented July 2, 1992, as a short communication at the conference "Operator Algebras 92" in Orléans, France, a satellite conference of the first European Congress of Mathematics, organized by the E.R.A. no. 747.

1991 Mathematics Subject Classification. Primary 22D25; Secondary 46L05, 43A07.

Key words and phrases. $C^{*}$-algebra, regular representation, amenability, nuclearity. 
The purpose of this note is to draw attention to $C^{*}(\lambda(G))$, the $C^{*}$-subalgebra of $\mathscr{B}\left(L^{2}(G)\right)$ generated by $\lambda(G)$. Of course, when $G$ is discrete, we have $C^{*}(\lambda(G))=C_{r}^{*}(G)$, and we will therefore mainly be interested in the nondiscrete case. In this case, it is known that $C_{r}^{*}(G)$ and $C^{*}(G)$ are nonunital [14, Corollaries 1 and 2], while $C^{*}(\lambda(G))$ is always unital.

The first paper we are aware of which explicitly deals with $C^{*}(\lambda(G))$ in the nondiscrete case is [12], where Kodaira and Kakutani essentially show that, when $G$ is abelian, $C^{*}(\lambda(G))$ is $*$-isomorphic to $\mathscr{C}\left(\widehat{G}_{d}\right)$, the continuous complex functions on the dual group of $G_{d}$. This result is nicely exposed by Arveson in [1], where he generalizes it to other $C^{*}$-algebras generated by abelian unitary groups. Further, when $G$ is abelian, it is well known that $C^{*}(G) \simeq C_{r}^{*}(G) \simeq$ $\mathscr{C}_{0}(\widehat{G})$, the continuous complex functions on the dual group of $G$ which vanish at infinity. Thus, $C^{*}(\lambda(G))$ on one hand and $C^{*}(G) \simeq C_{r}^{*}(G)$ on the other hand contain rather different information in the abelian case. Also, still in this case, we have $C_{r}^{*}\left(G_{d}\right) \simeq \mathscr{C}\left(\widehat{G}_{d}\right)$; hence, $C^{*}(\lambda(G)) \simeq C_{r}^{*}\left(G_{d}\right)$. Further, Zeller-Meier showed in [21] that the same conclusion is true whenever $G_{d}$ is amenable (see also $[3,4,9])$. One may therefore wonder whether all the topological flavour of $G$ always disappears in $C^{*}(\lambda(G))$.

We shall show that this suggestion is not generally true. Our approach relies heavily on the now well-developed theory of amenability $[17,18]$. We recall that $G$ is called amenable whenever there exists a left invariant mean on $L^{\infty}(G)$, i.e., a state on $L^{\infty}(G)$ which is invariant under left translations. A deep $C^{*}$ algebraic characterization of the amenability of $G$ is that $C^{*}(G)$ and $C_{r}^{*}(G)$ are canonically $*$-isomorphic ([17, Theorem 4.21] or [18, Theorem 8.9]). Another characterization via $C^{*}(\lambda(G))$ is possible: our first result (Theorem 1) is that $G$ is amenable if and only if there exists a nonzero multiplicative linear functional on $C^{*}(\lambda(G))$. We notice that the "only if" part is known in the discrete case [6, Theorem 2; 16, Proof of Proposition 1.6], and that, as pointed out to us by Alain Valette, a stronger result than Theorem 1 may be obtained (see the comments following Theorem 1). Our result provides a natural $C^{*}$ explanation to the fact that an abelian group $G$ is amenable: $C^{*}(\lambda(G))$ is then an abelian $C^{*}$-algebra and therefore possesses a nonzero multiplicative linear functional by Gelfand's theory. Of course, this is not the most efficient way to prove this fact, which is an easy consequence of the Markov-Kakutani fixed point theorem (cf. [17, Proposition 0.15]).

With the help of Theorem 1, we can complement the result of Zeller-Meier in [21] and conclude that $G_{d}$ is amenable if and only if $G$ is amenable and $C^{*}(\lambda(G)) \simeq C_{r}^{*}\left(G_{d}\right)$ (Theorem 3). Hence, if $G$ is an amenable group such that $G_{d}$ is not amenable (e.g., $\left.G=\mathrm{SO}(3)\right)$, then $C^{*}(\lambda(G))$ is not $*$-isomorphic to $C_{r}^{*}\left(G_{d}\right)$.

At last, we characterize the nuclearity of $C^{*}(\lambda(G))$. We recall that a $C^{*}$ algebra is called nuclear if there is a unique way of forming its tensor product with any other $C^{*}$-algebra. For some equivalent definitions, the reader may consult $[13,17,19]$, where further references are given. As a sample of the work of many hands, we quote the following from [17, 1.31 and 2.35]:

$G$ is amenable if and only if $G$ is inner amenable and $C_{r}^{*}(G)$ is nuclear, if and only if $G$ is inner amenable and $v N(G)$ is injective. 
Inner amenability of $G$ means here that there exists a state on $L^{\infty}(G)$ invariant under the action on $L^{\infty}(G)$ by inner automorphisms of $G$, while $v N(G)$ is injective whenever there exists a norm one projection from $\mathscr{B}\left(L^{2}(G)\right)$ onto $v N(G)$. We also recall that there exist nonamenable groups $G$ such that $C_{r}^{*}(G)$ is nuclear and $v N(G)$ is injective. Now, since any discrete group is inner amenable in the above sense, we have that $G_{d}$ is amenable if and only if $C_{r}^{*}\left(G_{d}\right)$ is nuclear, a result proved by Lance in [13, Theorem 4.2]. We shall use this to conclude that $G_{d}$ is amenable if and only if $C^{*}(\lambda(G)$ ) is nuclear (Theorem $3)$. Especially, we get that if $G$ is amenable but $G_{d}$ is not, then $C^{*}(\lambda(G))$ is nonnuclear while $C_{r}^{*}(G)$ is nuclear and $v N(G)$ is injective.

\section{THE RESULTS}

We first give a quite direct proof of the following result.

Theorem 1. $G$ is amenable if and only if there exists a nonzero multiplicative linear functional on $C^{*}(\lambda(G))$.

Proof. Suppose $G$ is amenable. Then there exists a net $\left\{\xi_{i}\right\}$ in $\left\{\xi \in L^{2}(G) \mid\|\xi\|_{2}\right.$ $=1\}$ such that

$$
\left\|\lambda(g) \xi_{i}-\xi_{i}\right\|_{2} \rightarrow 0 \text { for all } g \text { in } G
$$

(cf. [17, Theorem 4.4] or [18, Corollary 6.15]). For each $i$, define $\varphi_{i}$ on $C^{*}(\lambda(G))$ by

$$
\varphi_{i}(x)=\left\langle x \xi_{i}, \xi_{i}\right\rangle, \quad x \in C^{*}(\lambda(G)) .
$$

Then $\left\{\varphi_{i}\right\}$ is a net in the state space of $C^{*}(\lambda(G))$ which (by Banach-Alaoglu's theorem) is weak*-compact. Hence we may pick a weak*-limit point of this net, say $\varphi$, which is a state on $C^{*}(\lambda(G))$. Now, since

$$
\left|\varphi_{i}(\lambda(g))-1\right|=\left|\left\langle\left(\lambda(g) \xi_{i}-\xi_{i}\right), \xi_{i}\right\rangle\right| \leq\left\|\lambda(g) \xi_{i}-\xi_{i}\right\|_{2} \rightarrow 0 \text { for all } g \text { in } G,
$$

we clearly have $\varphi(\lambda(g))=1$ for all $g$ in $G$. From the Cauchy-Schwartz inequality for states, one then easily gets that $\varphi$ is multiplicative at each $\lambda(g)$, $g \in G$. As $\lambda(G)$ generates $C^{*}(\lambda(G))$ by definition, it follows by density that $\varphi$ is a nonzero multiplicative linear functional on $C^{*}(\lambda(G))$.

Conversely, suppose $\varphi$ is such a functional on $C^{*}(\lambda(G))$. Then, as $\varphi$ preserves adjoints [15, Proposition 2.1.9], $\varphi$ is a state on $C^{*}(\lambda(G))$ such that $|\varphi(\lambda(g))|=1$ for all $g$ in $G$. By the Hahn-Banach theorem for states [5, Proposition 2.3.24], we may extend $\varphi$ to a state $\tilde{\varphi}$ on $\mathscr{B}\left(L^{2}(G)\right)$ which satisfies

$$
|\tilde{\varphi}(\lambda(g))|=1 \text { for all } g \text { in } G \text {. }
$$

Again from the Cauchy-Schwartz inequality for states, it follows that $\tilde{\varphi}$ is multiplicative at each $\lambda(g), g \in G$.

We then have

$$
\begin{aligned}
\tilde{\varphi}\left(\lambda(g) x \lambda\left(g^{-1}\right)\right) & =\tilde{\varphi}(\lambda(g)) \tilde{\varphi}\left(x \lambda\left(g^{-1}\right)\right)=\tilde{\varphi}(\lambda(g)) \tilde{\varphi}(x) \tilde{\varphi}\left(\lambda\left(g^{-1}\right)\right) \\
& =|\tilde{\varphi}(\lambda(g))|^{2} \tilde{\varphi}(x)=\tilde{\varphi}(x)
\end{aligned}
$$

for all $g$ in $G$ and $x$ in $\mathscr{B}\left(L^{2}(G)\right)$.

The amenability of $G$ follows readily from this in a quite standard way (cf. [2, Theorem 2.2] or [6, Theorem 2]). If $M_{f}$ denotes the multiplication operator on $L^{2}(G)$ by $f \in L^{\infty}(G)$, then one obtains a left invariant mean $m$ 
on $L^{\infty}(G)$ by defining $m(f)=\tilde{\varphi}\left(M_{f}\right), f \in L^{\infty}(G)$, and by using that $M_{f_{g}}=$ $\lambda(g) M_{f} \lambda\left(g^{-1}\right)$ for all $f$ in $L^{\infty}(G)$ and $g$ in $G$, where $f_{g}(h)=f\left(g^{-1} h\right)$, $h \in G$.

When $U$ is a continuous unitary representation of $G$ on a Hilbert space $\mathscr{H}$, we denote by $\pi_{U}$ the canonically associated *-representation of $C^{*}(G)$ in $\mathscr{B}(\mathscr{H})$ (cf. [8]). We recall that if $V$ is another such representation of $G$, then $U$ is said to be weakly contained in $V$ (resp. weakly equivalent to $V$ ) whenever $\operatorname{ker} \pi_{V} \subseteq \operatorname{ker} \pi_{U}\left(\right.$ resp. $\left.\operatorname{ker} \pi_{V}=\operatorname{ker} \pi_{U}\right)$.

By regarding $G$ as a discrete group, we may consider $\lambda$ as a representation of $G_{d}$ in $L^{2}(G)$. To avoid confusion, we shall denote this representation by $\lambda^{\circ}$. It is then clear that $C^{*}(\lambda(G))=\pi_{\lambda^{\circ}}\left(C^{*}\left(G_{d}\right)\right)$.

In the above terminology, Theorem 1 (and its proof) may be reformulated as

Theorem $\mathbf{1}^{\prime}$. The following statements are equivalent:

(i) $G$ is amenable.

(ii) The trivial one-dimensional representation of $G_{d}$ is weakly contained in $\lambda^{\circ}$.

(iii) There exists a character on $G_{d}$ which is weakly contained in $\lambda^{\circ}$.

As pointed out to us by A. Valette, the next statement may also be added:

(iv) There exists a finite-dimensional unitary representation of $G_{d}$ which is weakly contained in $\lambda^{\circ}$.

Of course, (iii) $\Rightarrow$ (iv) is trivial, while (iv) $\Rightarrow$ (ii) may be deduced from some results of Fell [8,13.11.3 and 18.9.15]. Alternatively, (iv) $\Rightarrow$ (i) may be derived from the work of Bekka in [2].

The next lemma plays an important role in the proof of Theorem 3 and is recorded here for the convenience of the reader.

Lemma 2. $\lambda_{d}$ is weakly contained in $\lambda^{\circ}$. Further, if $G_{d}$ is amenable, then $\lambda_{d}$ is weakly equivalent to $\lambda^{\circ}$, and $C^{*}(\lambda(G))$ is *-isomorphic to $C_{r}^{*}\left(G_{d}\right)$.

The first part of Lemma 2 is essentially known and follows immediately by combining the argument given in [1, p. 206] with [10, Theorem 1]. The crucial point is the existence of a net of positive definite functions on $G_{d}$ associated to $\lambda^{\circ}$ which converges pointwisely to the characteristic function of the identity element in $G_{d}$, a fact which is also established in the proof of [4, Proposition 1]. The second part of Lemma 2, whose last assertion is shown by Zeller-Meier in [21, Corollary 6] (see also [3, 1.13.ii; 9, Proposition 3.4]), follows now from the fact that, if $G_{d}$ is amenable, then $\lambda_{d}$ weakly contains all unitary representations of $G_{d}$ (cf. [8, 18.3.5 and 18.3.6]).

Theorem 3. The following statements are equivalent:

(i) $G_{d}$ is amenable.

(ii) $G$ is amenable, and $C^{*}(\lambda(G)) \simeq C_{r}^{*}\left(G_{d}\right)$.

(iii) $C^{*}(\lambda(G))$ is nuclear.

(iv) $C_{r}^{*}\left(G_{d}\right)$ is nuclear.

Proof. (i) $\Leftrightarrow$ (iv) is proved by Lance in [13, Theorem 4.2].

(i) $\Rightarrow$ (ii): Suppose $G_{d}$ is amenable. Then $G$ is amenable ([17, Problem 1.12] or [18, Proposition 4.21]) and $C^{*}(\lambda(G)) \simeq C_{r}^{*}\left(G_{d}\right)$ by Lemma 2 . 
(ii) $\Rightarrow$ (i): Suppose $G$ is amenable and $C^{*}(\lambda(G)) \simeq C_{r}^{*}\left(G_{d}\right)$. From Theorem 1, we then know that $C^{*}(\lambda(G))$ possesses a nonzero multiplicative linear functional and, therefore, that $C_{r}^{*}\left(G_{d}\right)$ possesses one too. Since $C_{r}^{*}\left(G_{d}\right)=$ $C^{*}\left(\lambda_{d}\left(G_{d}\right)\right)$, Theorem 1 now implies that $G_{d}$ is amenable.

(iii) $\Rightarrow$ (iv): Suppose $C^{*}(\lambda(G))$ is nuclear. Since $\lambda_{d}$ is weakly contained in $\lambda^{\circ}$ by Lemma 2 , this implies that $\pi_{\lambda_{d}}\left(C^{*}\left(G_{d}\right)\right)=C_{r}^{*}\left(G_{d}\right)$ is a quotient $C^{*}$ algebra of $\pi_{\lambda^{\circ}}\left(C^{*}\left(G_{d}\right)\right)=C^{*}(\lambda(G))$. As it is known that a quotient $C^{*}$-algebra of a nuclear $C^{*}$-algebra is itself nuclear [7, Corollary 4], we obtain that $C_{r}^{*}\left(G_{d}\right)$ is nuclear.

(iv) $\Rightarrow$ (iii): Suppose $C_{r}^{*}\left(G_{d}\right)$ is nuclear. Since we now know that (iv) $\Rightarrow$ (ii), we have $C^{*}(\lambda(G)) \simeq C_{r}^{*}\left(G_{d}\right)$, so $C^{*}(\lambda(G))$ is nuclear too.

We conclude this note with some remarks on

$$
X(G)=\left\{\varphi: C^{*}(\lambda(G)) \rightarrow \mathbf{C} \mid \varphi \text { is nonzero, linear, and multiplicative }\right\},
$$

which is a weak*-closed subset of the state space of $C^{*}(\lambda(G))$. Theorem 1 says that $X(G) \neq \varnothing$ if and only if $G$ is amenable. When $G$ is abelian, the result of Kodaira and Kakutani mentioned in the introduction may be interpreted as the fact that $X(G)$ is homeomorphic to $\widehat{G}_{d}$. In the nonabelian case, $X(G)$ is of course a rather primitive $C^{*}$-algebra invariant for $C^{*}(\lambda(G))$, but it has the advantage of being easily computed in some cases, as the following illustrates.

Let $H$ denote a discrete group and $\mathrm{CH}$ its commutator subgroup. Then $\mathrm{H} / \mathrm{CH}$ is abelian, and it is not difficult to show, as it has been observed by Watatani in [20], that if $H$ is amenable then $X(H)$ is homeomorphic to $\widehat{H / C} H$. Hence, if $G_{d}$ is amenable, via Theorem 2 we get that $X(G)$ is homeomorphic to $G_{d} \widehat{/ C} G_{d}$. If $G$ is amenable but $G_{d}$ is not, one can show that $X(G)$ contains a copy of $\widehat{G / \bar{C} G}$ and may itself be embedded as a subgroup of $G_{d} \widehat{/ C} G_{d}$, but we do not know whether anything more general can be said here. If, for example, $G=\operatorname{SO}(3)$, then $C G_{d}=G_{d}$, so $X(G)=\{\hat{1}\}$ (where $\hat{1}$ denotes the state on $C^{*}(\lambda(G))$ determined by $\hat{1}(\lambda(g))=1$ for all $g$ in $G$; cf. the proof of Theorem 1).

\section{ACKNOWLEDGMENTS}

It is a pleasure to thank Alain Valette and the referee for their helpful comments after reading the first draft of this paper. We are also indebted to Alain Valette for drawing our attention to references $[2,3,4,9]$.

\section{REFERENCES}

1. W. B. Arveson, $A$ theorem on the action of abelian unitary groups, Pacific J. Math. 16 (1966), 205-212.

2. M. Bekka, Amenable unitary representations of locally compact groups, Invent. Math. $\mathbf{1 0 0}$ (1990), 383-401.

3. M. Bekka, A. Lau, and G. Schlichting, On invariant subalgebras of the Fourier-Stieljes algebra of a locally compact group, Math. Ann. 294 (1992), 513-522.

4. M. Bekka and A. Valette, On duals of Lie groups made discrete, preprint, 1991.

5. O. Bratteli and D. W. Robinson, Operator algebras and quantum statistical mechanics, I, Springer-Verlag, New York, 1979. 
6. H. Choda and M. Choda, Fullness, simplicity and inner amenability, Math. Japon. 24 (1979), 235-246.

7. M. D. Choi and E. G. Effros, Nuclear $C^{*}$-algebras and injectivity; the general case, Indiana Univ. Math. J. 26 (1977), 443-446.

8. J. Dixmier, Les $C^{*}$-algèbres et leurs représentations (2ème ed.), Gauthier-Villars, Paris, 1969.

9. C. Dunkl and D. Ramirez, $C^{*}$-algebras generated by Fourier-Stieljes transforms, Trans. Amer. Math. Soc. 164 (1972), 435-441.

10. A. Figà-Talamanca, On the action of unitary groups on a Hilbert space, Symposia Math. 22 (1977), 314-319.

11. E. Hewitt and K. A. Ross, Abstract harmonic analysis, vol. I, Springer-Verlag, Berlin, Göttingen, and Heidelberg, 1963.

12. K. Kodaira and S. Kakutani, Normed ring of a locally compact abelian group, Proc. Imp. Acad. Tokyo 19 (1943), 360-365.

13. E. C. Lance, On nuclear $C^{*}$-algebras, J. Funct. Anal. 12 (1973), 157-176.

14. P. Milnes, Identities of group algebras, Proc. Amer. Math. Soc. 29 (1971), 421-422.

15. G. Murphy, Operator theory and $C^{*}$-algebras, Academic Press, New York, 1990.

16. W. L. Paschke and N. Salinas, $C^{*}$-algebras associated with the free product of groups, Pacific J. Math. 82 (1979), 211-221.

17. A. L. Paterson, Amenability, Math. Surveys Monographs, vol. 29, Amer. Math. Soc., Providence, RI, 1988.

18. J. P. Pier, Amenable locally compact groups, Wiley, New York, 1984.

19. A. M. Torpe, Notes on nuclear $C^{*}$-algebras and injective von Neumann algebras, preprint, Mat. Inst., Odense Universitet, 1981.

20. Y. Watatani, The character group of amenable group $C^{*}$-algebras, Math. Japon. 24 (1979), 141-144.

21. G. Zeller-Meier, Représentations fidèles des produits croisés, C. R. Acad. Sci. Paris Sér. A 264 (1967), 679-682.

Institute of Mathematics, University of Oslo, P.B. 1053 Blindern, 0316 Oslo 3, NoRWAY

E-mail address: bedos@math.uio.no 